\title{
Téoros
}

Revue de recherche en tourisme

\section{Le transport aérien entre le Québec et la France}

\section{Paul Simier}

Volume 8, numéro 1, mars 1989

France-Québec

URI : https://id.erudit.org/iderudit/1080358ar

DOI : https://doi.org/10.7202/1080358ar

Aller au sommaire du numéro

Éditeur(s)

Université du Québec à Montréal

ISSN

0712-8657 (imprimé)

1923-2705 (numérique)

Découvrir la revue

Citer cet article

Simier, P. (1989). Le transport aérien entre le Québec et la France. Téoros, 8(1), 33-40. https://doi.org/10.7202/1080358ar d'utilisation que vous pouvez consulter en ligne.

https://apropos.erudit.org/fr/usagers/politique-dutilisation/ 


\section{Le transport aérien entre le Québec et la France}

Paul Simier*

\section{Le marché se réglemente très bien de lui-même. De quoi se mêlent Paris et Ottawa avec des quotas?}

Bon an mal an, 250000 Canadiens, dont une forte majorité de Québécois, franchissent l'Atlantique pour aller découvrir "le vieux pays" en commençant leur périple par la France. Le mouvement, loin de s'essouffler. repart de plus belle chaque année. La soif du retour aux sources est évidement toujours présente mais c'est surtout la sollicitation, à coups de bas tarifs, que les compagnies aériennes pratiquent sur le public qui explique cet engouement. L'attrait que le Québec exerce sur les Français se concrétise aussi, si l'on en croit la croissance du tourisme réceptif dans la Belle Province. Paradoxalement, les gouvernements, tant français que canadien, à qui tout cela profite, tendent àt vouloir réglementer lé transport aćrien entre les deux pays.

Les voyageurs aussi bien québécois que français doivent leur salut à une compagnie qu'une offre publique d'achat vient de faire taire au mois de janvier 1989. N'eût été, en effet de petite compagnie aérienne privée mais aussi le plus beau fleuron de tous les transporteurs qu'ait comptés le pays, le voyage transatlantique par avion serait encore aujourd'hui l'apanage de gens aisés, sinon d'une élite.

Les deux compagnies aériennes régulières nationales, Air Canada et Air France, planifiaient alors de fixer à près de 1000 dollars le plus bas tarif entre Montréal et Paris quand Wardair, alors simple transporteur charter, vint fausser sérieusement le jeu concerté des deux grands, ramenant les tarifs à des niveaux beaucoup plus abordables.

\section{Les lois du marché}

C'était il y a sept ou huit ans. Le dernier arrivé, taxé de vouloir écrémer le marché durant les saisons les plus propices, eut raison des vélléités des deux compagnies régulières.

Depuis cette époque, les tarifs décents sont restés de mise avec même une forte tendance à la stabilité du fait de la multiplication des transporteurs nolisés sur cette route. Depuis cinq ans, trois autres transporteurs aériens

\footnotetext{
* Paul Simier est chroniqueur en tourisme et chargé de
} cours à l'Université de Québec à Montréal à la demande (Minerve Canada, Nationair et Transat), tous basés à Montréal, ont vu le jour avec pour objectif de tirer leurs bénéfices des flux de vacanciers ćtablis sur l'Atlantique nord, en plus des destinations soleil durant l'hiver.

La surenchère a eu pour résultat de maintenir des tarifs bas et même parfois surprenants. La concurrence fut très vive d'ailleurs entre les transporteurs charters eux-mèmes, on en vit deux se disputer pour maintenir une liaison hebdomadaire même au plus creux de la saison hivernale. Du coup, les récriminations des transporteurs réguliers et l'argument de l'écrémage du marché en haute saison devenaient caducs.

Conséquence de la forte concurrence des dernières années: le billet Montréal-Paris allerretour coûte ainsi moins cher, même en haute saison, que celui que doit payer un voyageur venant des îles de la Madeleine ou de Gaspésie à Montréal pour prendre son vol transatlantique. La logique des règles de la libre entreprise s'est établie et semblait faire l'affaire de tous, à commencer par les voyageurs eux-mêmes.

\section{Des effets favorables}

Les effets de l'agressivité des compagnies aériennes spécialisées dans le nolisement, et dans la foulée des voyagistes, se sont fait sentir très favorablement. En France, les prestataires de services, hôteliers et loueurs d'autos, en attestent régulièrement en publiant les statistiques concernant la progression de leur chiffre d'affaires généré par les touristes québécois.

Au Québec, villes et régions se félicitent de la hausse constante du nombre de touristes français attirés notamment par des tarifs aćriens plus favorables, donc par un cout de voyage plus abordable.

L'industrie du voyage a appris à vivre avec la nouvelle situation. Au lieu de vendre cher quelques billets d'avion, on a découvert qu'on pouvait multiplier le nombre de clients en vendant à bas prix et en faisant plus de volume.

\section{Des quotas}

On aurait pu croire les règles enfin établies. et les vieux démons du protectionnisme repoussés à jamais. En réalité, le spectre de la réglementation à outrance n'a jamais cessé de planer dans le dossier du transport aérien entre le Québec et la France.
Tout au long de ces années où l'on a vu les charters livrer bataille et contrer les deux compagnies régulières tant sur le terrain des prix que sur celui du service, les pressions n'ont cessé de la part des gouvernements. On est ainsi passé par toutes les phases et toutes les subtilités. Une année, le Gouvernement français, par le biais de sa Direction générale de l'aviation civile (D.G.A.C.), voulait amener les transporteurs nolisés canadiens à respecter un tarif minimum; l'année suivante, on leur refusait le droit d'atterrir sous le prétexte que trop de vols battaient pavillon canadien.

La guéguerre n'a cessé tout ce temps. Cette guérilla fut toujours le fait de la D.G.A.C. dont les responsables, en réunion publique à Paris, ne se cachaient même pas que leur devoir était de protéger les intérêts commerciaux (et les tarifs) de leur compagnie nationale, Air France.

On aura ainsi vu des vols Montréal-Paris détournés au dernier moment sur Bruxelles, à défaut de droit d'atterrir. Pour jeter le discrédit sur une compagnie et ébranler la crédibilité des petits transporteurs dans l'opinion on ne pouvait trouver de meilleur instrument... Ce que la France tentait depuis toujours, limiter le nombre de vols pour tenter de faire grimper les tarifs, a finalement pris la forme de quotas, imposés de concert avec le gouvernement d'Ottawa pour trois ans.

Au nom du règlement ainsi adopté bilatéralement, le nombre de sièges concédés aux transporteurs nolisés est plafonné à 200000 sur l'aller-retour pour les compagnies canadiennes. Par contre, les transporteurs français ne sont pas visés par cette mesure. De plus, en basse saison, de l'automne à la fin du printemps, sauf durant les périodes de Noël et de Páques, la fréquence des vols nolisés assurés par des transporteurs canadiens est limitée à une liaison. Ceci écarte done du marché deux compagnies aériennes sur trois dans cette catégorie durant près de six mois.

On dit que le gouvernement d Ottawa a accepté ces quotas contre les droits accordés à Wardair pour desservir sur une base régulière Paris et Lyon au départ du Canada... Au début de cette année, le responsable de l'une des compagnies de nolisement canadiennes affirmait que I'application à la lettre des quotas aurait pour effet de faire croitre

(Suite à la page 40) 
à 15.000 mois de stage par an, privilégiant les agences de voyages, les villages de vacances. De ce fait, il y a interaction entre les stages et l'emploi saisonnier, de nombreuses possibilités de "dérapage" entre la notion de stagiaite et de saisonnier. Et puis il y a vraisemblablement les " $B O N S$ " et les "MONS BONS" stagiaires: Pour reprendre l'exemple du BTS de Tourisme, il ne faut pas négliger le fait qu'en fin de première année de cours, donc au milieu d'un cycle non achevé, de 60 à $70 \%$ des stages sont effectués par des stagiaires qui échoueront à l'examen; ceci pose un problème d'image pour la formation en elle-même auprès des entreprises et maitres de stages.

Les particularités des entreprises touristiques et de la forte saisonnalité de l'activité en France peuvent être à l'origine de quelques inconvénients:

- présence simultance de trop nombreux stagiaires dans une petite entreprise;

- niveau d'activité trop, ou trop peu, élevé pendant les vacances scolaires où se déroulent la plupart des stages, suivant que l'entreprise se trouve en zone émettrice ou en zone d'accueil touristique;

- sous-représentation de certaines catégories d'entreprises inhérente à la saison estivale qui est celle des stages: secteur des loisirs urbains... et par définition ignorance totale de l'activité sports d'hiver!

- dans certains cas, indifférence réciproque des stagiaires et des entreprises, le stage étant vécu comme une obligation pour les uns, un service rendu à des établissements scolaires pour les autres.

Comment améliorer globalement l'exploitation pédagogique du stage au cours d'un cycle de formation? Plusieurs pistes peuvent être explorées, certaines le sont déjà:

- des stages assortis à un projet dans l'entreprise, que ce soit dans le cadre d'une réflexion globale sur la place d'un stagiaire dans l'entreprise ou d'un projet précis (enquête à mener ou autre...);

- un encadrement et un suivi du centre de formation pendant le stage, pouvant se traduire par des visites, lesquelles contribuent toujours à améliorer la communication entre formateurs et entreprises;

- des accords contractuels ne se limitant pas au statut du stagiaire mais s"étendant à son insertion, à une "charte de qualite" du stage;

- des stages non coupés du reste de la formation (stages en cours de cycle et non à la fin, stages éventuellement fragmentês, etc.);

- un véritable mémoire de stage qui pourrait être inséré dans une recherche individuelle du stagiaire, un projet précis et ceci quel que soit le niveau de formation, une telle démarche n'étant pas une exclusivité de l'université;
- des stages ne se limitant pas au territoire national mais s'étendant à l'Europe. Le problème de l'encadrement des stagiaires pourrait êttre réglé par des "échanges de suivi des stages " avec des établissements étrangers: la coopération en ce domaine pourrait être fructueuse.

Ces remarques vont dans le sens d'une plus grande importance accordée aux stages, laquelle va de pair avec une plus grande attention accordée à leur finalité et aux modalités de leur déroulement.

Une réflexion des formateurs sur les moyens disponibles aujourd hui ne doit pas dispenser d'une démarche similaire quant à la finalité des formations et à leurs résultats. Elle ne peut non plus êttre menée isolément, coupée des autres catégories d'intéressés: ainsi, il faudra bien progresser en direction d'une confiance mutuelle dans les fonctions des formateurs et milieux professionnels du tourisme et des loisirs. Chacun a besoin des autres et si les formations intégrées à l'entreprise restent l'apanage des grands groupes, même ceux-ci ont besoin de futurs collaborateurs bénéficiant d'une bonne formation initiale.

La formulation précise des constats aide a celle des propositions, c'est une évidence, mais une barrière sépare toujours ces deux niveaux: celle justement qui est constituée par les moyens disponibles. La faiblesse de ceux-ci rend a priori certaines propositions "irréalistes" et leur formulation devient inutile: pourquoi, alors, mener une réflexion?

Mais si les formateurs ne réflechissent pas individuellement et collectivement sur leur métier, les risques de déphasage sont grands d'autant plus que les evvolutions intervenant dans leurs activités proviennent quelquefois de l'extérieur et sont difficilement perceptibles, Ainsi, les "Dossiers de l'Éndiant" jouent un certain rôle dans le marché de la formation touristique en publiant chaque année les résultats d'établissements proposant des candidats aux examens débouchant sur des BTS du tertiaire, dont le BTS Tourisme. Cette transparence de l'information est forcément positive; avec quels résultats? Moralisation du marché? Développement d'une attitude de consommateurs de formation? En tout cas, le problème des grilles d'évaluation des formations est posé.

La coupure entre formation initiale et continue doit aussi faire l'objet d'une réflexion: l'obtention d'un diplôme de type " $b a c+2$ ", "bac +3 ", etc... ne peut pas constituer pour un individu l'investissement-formation définitif sur lequel il va asseoir sa carrière. Dans un secteur où la taille des entreprises est réduite et la mobilité des actifs est élevée. il faut aussi que la demande en formation continue vienne des intéressés, qu'ils gèrent leur carrière en intégrant la composante FORMATION, Comment les y aider? L'émiettement des offres de stages n'est pas forcément sécurisant pour cela. Pourquoi ne pourrait-on pas concevoir une formation continue composée d'unités capitalisables acquises

- dans des centres publics ou assimilés,

- dans des centres privés agréés avec des formations reconnues valables, et ceci sur l'ensemble du territoire national, puis européen?

Les moyens existent, leur seule mise en oeuvre cohérente est nécessaire.

Dernier thème de réflexion pour cet article: la nature des emplois créés par certains secteurs du tourisme et des loisirs. N'oublions pas que les terrains de golf génèrent de nombreux emplois de jardiniers et que les parcs de loisirs ont besoin d" "hommesorchestres" de l'animation. Le tourisme ne créant pas que des emplois "touristiques". il est indispensable d'identifier les emplois pour lesquels les formations touristiques peuvent réllement faire quelque chose.

\begin{abstract}
Références
I.N.S.E.E. Institut National de la Statistique et des Etudes Economiques]: dossier L'entrée des jeunes dans la vie active, Economie et Statistiques no 216 , decembre 1988, p. 23 aे 59.

LEHALLLE Dominique; European Institute of Tourism Management - La grande 6 cole, Echo Touristique no 40. 21 novembre 1988

ROUGE Marianne: Les L.E.A. jouent l'Europe, Le Monde, 2 février 1989

RUFFIN Françoise: La diversité des formations aux métiers du tourisme, collogue L'enjeu touristique, Ed. Economca, 1988, р. 259 a 266 .
\end{abstract}

\section{(Suite de la page 33 )}

les tarifs de 20 à $25 \%$ entre le Québec et lat France.

\section{Gouvernement muet}

Les autorités gouvernementales québécoises sont restées muettes dans ce dossier. Pourtant, le fait de limiter l'offre risque de faire monter les prix. Pour qui se préoccupe de réduire le déficit de la balance des échanges touristiques, une telle mesure est positive parce que de nature à dissuader les gens de se rendre en Europe. Tout aussi logiquement, ceci pourrait refroidir les ardeurs des Français dans leur désir de venir sillonner le Québec. Les années qui viennent seront donc décisives en ce qui concerne l'aboutissement de ce dossier. Les velléités protectionnistes de la France sont connues en matière de transport aérien, mềne si elles sont souvent contraires aux intérêts de son tourisme.

A l'inverse, la politique de déréglementation d'Ottawa - à l'intérieur d'abord puis répercutée dans le même esprit dans les relations bilatérales - semblait tout aussi évidente. Mais il va falloir attendre entre deux ans pour s'en convaincre que prennent fin les règlements actuels régissant les vols CanadaFrance. 